\title{
Successful live birth after heterotopic ruptured cornual pregnancy with twin intrauterine gestation in an in vitro fertilization conception
}

\author{
Venus Bansal $^{1}$, Muskaan Chhabra ${ }^{1 *}$, Rahul Chopra² ${ }^{2}$ Pooja Prajapati ${ }^{1}$
}

\author{
${ }^{1}$ Department of Obstetrics and Gynecology, SPS Hospitals, Ludhiana, Punjab, India \\ ${ }^{2}$ Department of Radiology, SPS Hospitals, Ludhiana, Punjab, India
}

Received: 27 September 2019

Accepted: 12 November 2019

\section{*Correspondence:}

Dr. Muskaan Chhabra,

E-mail: muskan.chh@gmail.com

Copyright: (C) the author(s), publisher and licensee Medip Academy. This is an open-access article distributed under the terms of the Creative Commons Attribution Non-Commercial License, which permits unrestricted non-commercial use, distribution, and reproduction in any medium, provided the original work is properly cited.

\begin{abstract}
Heterotopic pregnancies, especially in the cornual region which were a rarity till recent times, have become a more common occurrence due to increasing practice of assisted reproduction. Optimal management of such cases is imperative to manage the risk of hypotension and shock in case of rupture and to judiciously preserve the intrauterine pregnancy. Here we are reporting a case of IVF conception of twin intrauterine pregnancy with a cornual heterotopic pregnancy. Patient presented to the emergency department with features of acute abdomen, haemoperitoneum and shock. Ultrasound findings were suggestive of rupture of cornual heterotopic pregnancy, $1500 \mathrm{ml}$ haemoperitoneum with live twin intrauterine gestation. Patient was resuscitated with iv fluids blood transfusion. Immediate laparotomy was done and cornual site repaired and covered with an omental patch while preserving the intrauterine gestations. Thereafter, pregnancy was carefully monitored with a high index of suspicion for rupture of site of cornual repair with advancing gestational age. Patient was readmitted at 24 weeks with pain abdomen and cornual site was found to be $4 \mathrm{~mm}$ in thickness. She was managed conservatively till 27 weeks when she had preterm rupture of membranes and emergency LSCS was done. She delivered healthy twin male babies, 780 gmb and 795 gm respectively. This case demonstrates that cornual heterotopic pregnancy is a diagnosis which may be easily missed and can present as a lifethreatening complication if it ruptures and significant intraperitoneal bleeding occurs. However, it is possible to successfully manage these cases with timely intervention, proper uterine reconstruction and monitoring of intrauterine gestation.
\end{abstract}

Keywords: Beta human chorionic gonadotropin, Cornual, Heterotopic, In vitro fertilization, Rupture

\section{INTRODUCTION}

Heterotopic pregnancy is defined as coexistence of intrauterine with extrauterine gestation. A naturally occurring heterotopic pregnancy is a rarity with incidence being as low as 1 in $30,000 .{ }^{1}$ But with the advent of assisted reproductive techniques, the scenario has radically changed. With the increased availability of In vitro fertilization techniques, heterotopic pregnancies are more commonly encountered today and the incidence of these in IVF pregnancies shoots up to 1 in 100 to 1 in $500 .^{2}$ However, the management protocols of such pregnancies may vary greatly depending upon the location of the intrauterine pregnancy, hemodynamic stability of the patient and the gestation of the pregnancy. We present a case report detailing one such patient where a heterotropic cornual pregnancy was seen along with an intrauterine twin pregnancy and was successfully managed. 


\section{CASE REPORT}

Patient was 33 years old Primigravida who had undergone multiple trials of assisted reproduction. She had two previous abortions that were surgically terminated followed by 3 cycles of IVF, of which two were unsuccessful and the last one resulted in a ruptured ectopic pregnancy for which left salpingectomy was done. She conceived her present pregnancy in her fourth attempt of IVF.

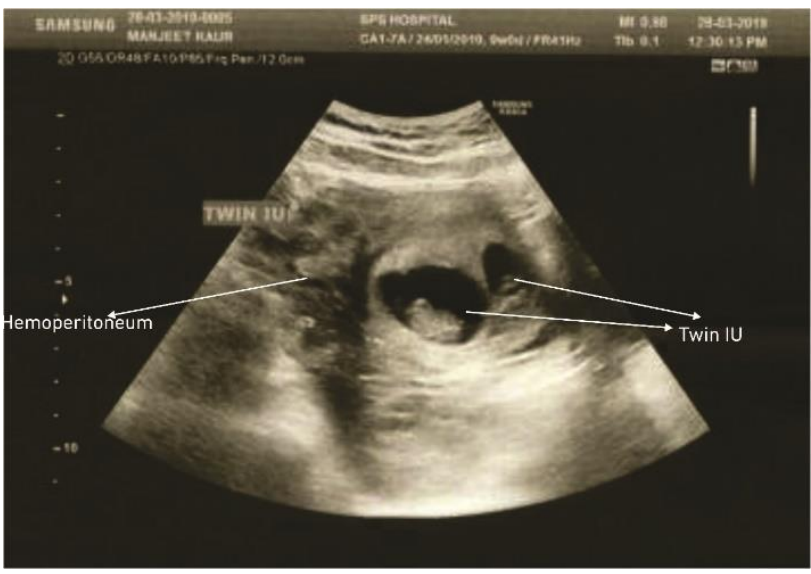

Figure 1: Ruptured cornual heterotopic pregnancy with Intrauterine twin pregnancy with haemoperitoneum.

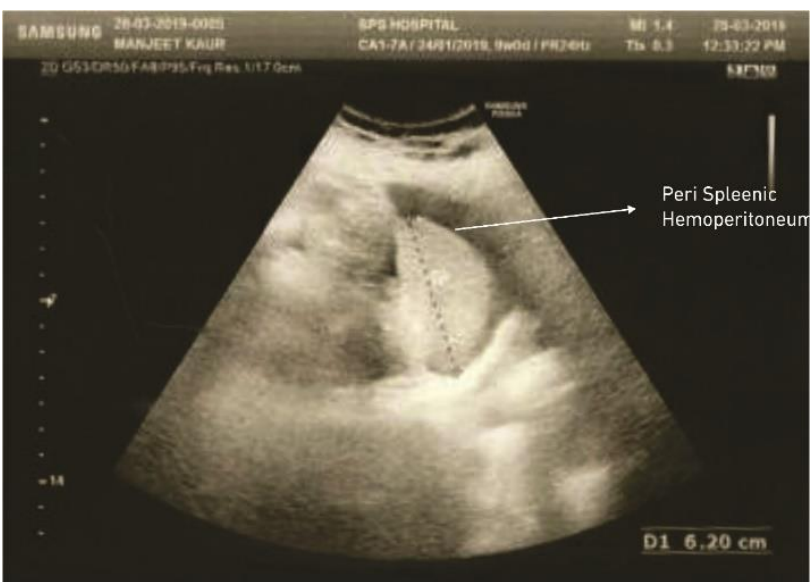

Figure 2: Perisplenic haemoperitoneum.

Patient was received in emergency department of our unit referred from the above-mentioned IVF centre, with a USG suggestive of dichorionic diamniotic twin intrauterine gestation of 8 weeks along with ruptured left cornual ectopic pregnancy with haemoperitoneum. The cornual pregnancy was seen to have developed at the side of previous salpingectomy. On admission, she was haemodynamically unstable with a pulse of 120 beats/min, thread, low volume and a BP of $90 / 70 \mathrm{~mm} \mathrm{Hg}$. Abdomen was distended, tense, tender and fluid thrill was present. Patient was clinically pale with haemoglobin of $9.4 \mathrm{gm} / \mathrm{dl}$ and complaining of severe pain abdomen. Resuscitation of the patient was started with IV fluids and blood was arranged. A repeat ultrasonography was done to evaluate the fetal heartbeat of the intrauterine twin pregnancy (Figure 1-3). Patient was taken to OT after informed consent and High risk of loss of intrauterine pregnancy explained. Exploratory laparotomy followed by drainage of haemoperitoneum and repair of ruptured cornu with securing haemostasis planned. Progesterone support and tocolysis were given.

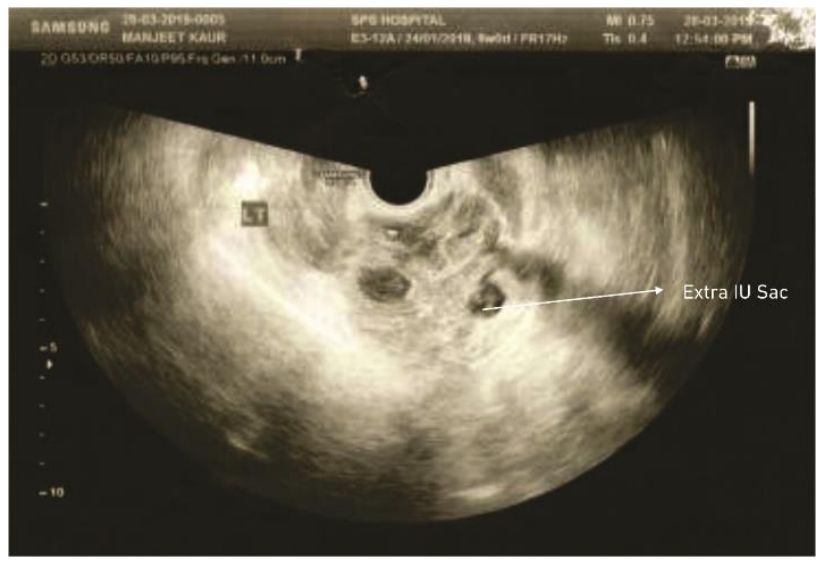

Figure 3: Extrauterine sac with haemoperitoneum.

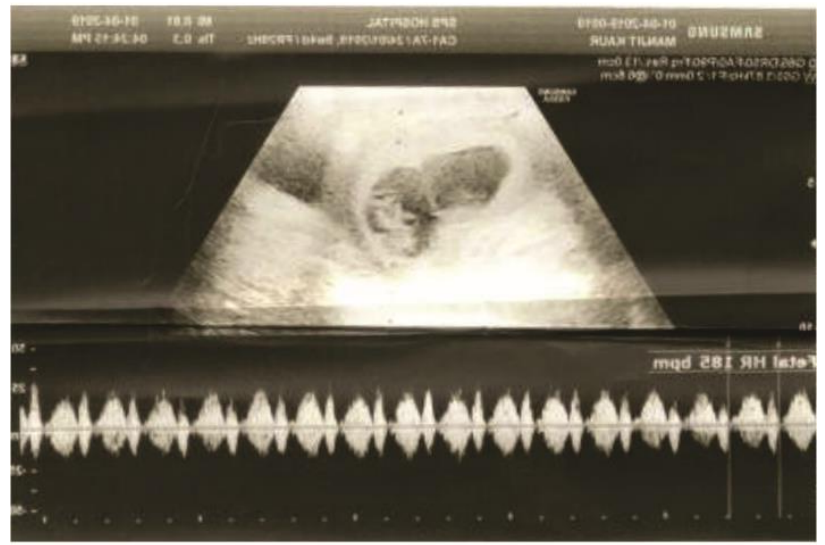

Figure 4: Post laparotomy intrauterine twin pregnancy with fetal cardiac activity.

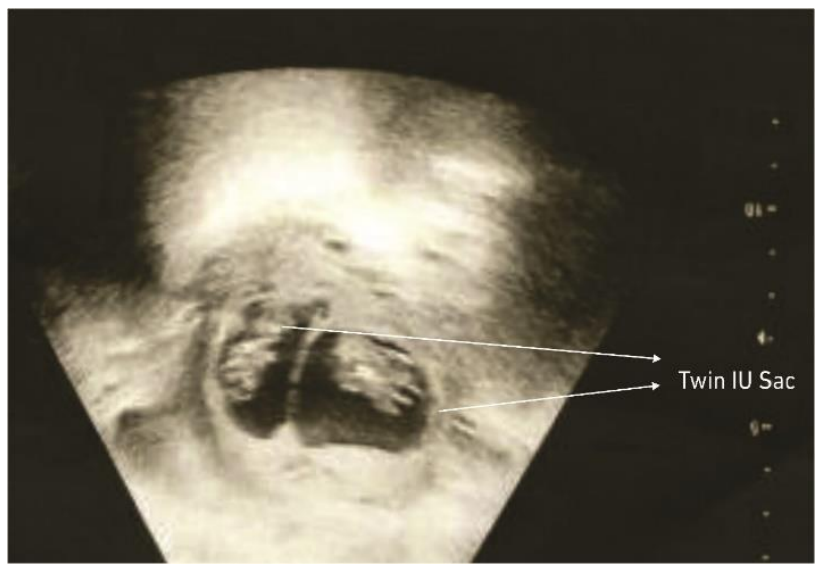

Figure 5: Post laparotomy live twin intrauterine pregnancy just prior to discharge. 


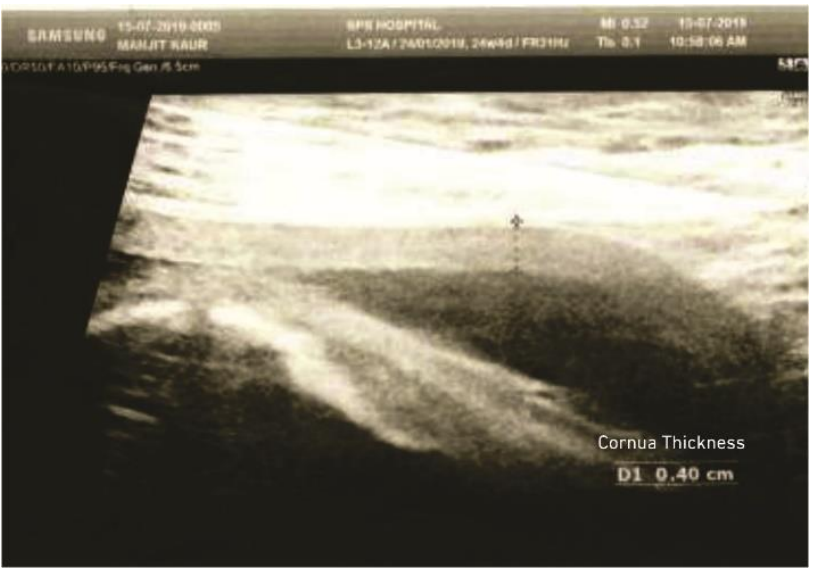

Figure 6: Cornual thickness at 24 weeks of gestation $4 \mathrm{~mm}$.

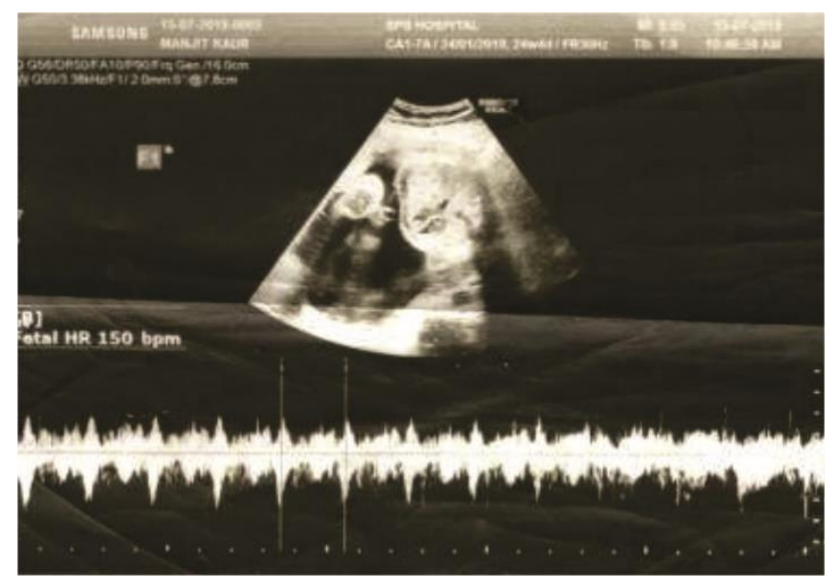

Figure 7: Intrauterine twin 1 with cardiac activity.

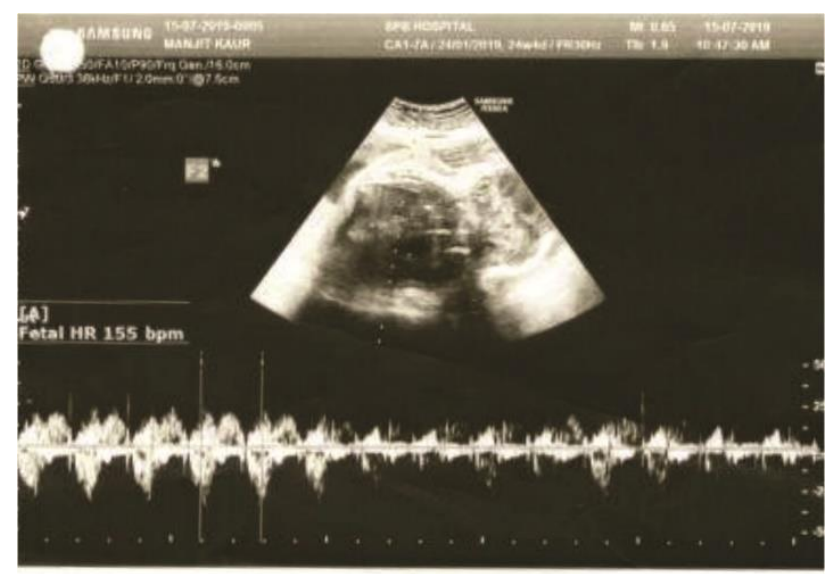

Figure 8: Intrauterine twin 2 with cardiac activity.

Intraoperatively uterus was gravid, 12 weeks in size. Ruptured left cornual pregnancy was seen with haemoperitoneum of $1500 \mathrm{ml}$ with clots. The cornual pregnancy was carefully excised and bleeding controlled from cornual site. A very thin layer of myometrium was present over the intrauterine pregnancy at the cornual site and bulging of membranes felt through it. Sutures were taken with polygalactone 20 in two layers and haemostasis secured. An omental patch was made and cornual site covered. Surgery on pregnant uterus with twins leading to a very soft, friable and vascular myometrium was a difficult. Moreover, the risk of delayed healing and rupture before healing (which could be catastrophic) was a major concern. A large haemoperitoneum can be the cause of hypoxia for the intrauterine viable pregnancy. Haemoperitoneum drained and peritoneal wash was given. One-unit PRBC transfused. Fetal heart beats of twin intrauterine pregnancy were ascertained immediately postoperatively and before discharge (Figure 4, 5). Patient was sent home in a stable condition with live intrauterine pregnancy and asked to maintain regular follow-up.

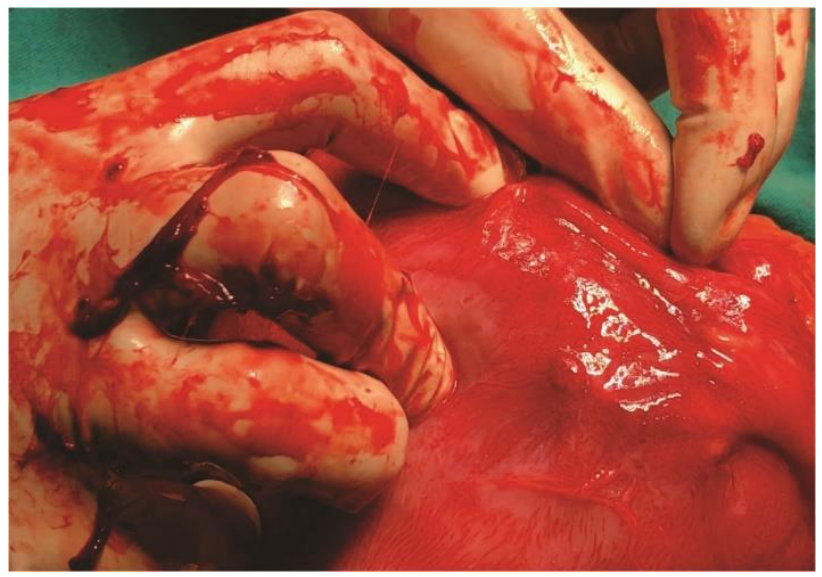

Figure 9: Cornual dimple seen during LSCS.

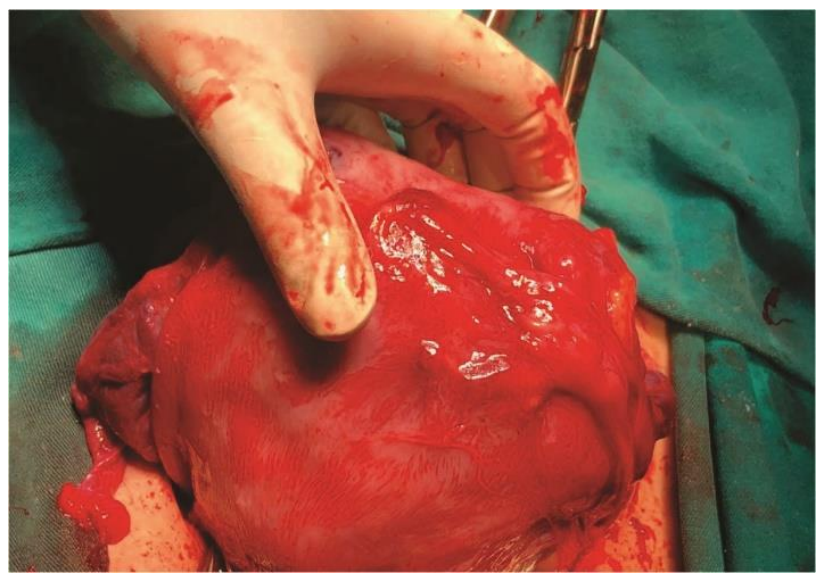

Figure 10: Intraoperative healed uterus as seen during LSCS.

Patient was monitored with regular antenatal surveillance till 24 weeks of gestation over the course of which she developed gestational Diabetes and was started on Inj. Insulin and diet control. At 24 weeks she was readmitted with complaints of pain in lower back and abdomen. A repeat ultrasound was done to assess the cornual scar site for integrity and thickness. On ultrasound the intrauterine twin babies showed a discordance of $8 \%$ and myometrial thickness over the left cornu was $4 \mathrm{~mm}$ (Figure 6-8). She was started on conservative treatment with antenatal fetal 
surveillance, progesterone support, antenatal steroid cover and management of GDM. Patient had a pulse rate of 130 to 140 beats per minute on admission which was evaluated with ECG and echocardiography and was found to be sinus tachycardia. Monitoring for detection of a possible rupture became difficult due to tachycardia. At 27 weeks gestation, patient complained of leaking PV in the ward. She was taken for emergency LSCS under spinal anaesthesia in view of PROM and preterm labour. Intraoperatively, cornual region was thin but untact. (Figure 9, 10). Twin male babies were delivered, 780gm and $795 \mathrm{gm}$ and shifted to NICU. After 64 days, babies discharged home at a weight of $1.8 \mathrm{~kg}$ and $1.425 \mathrm{~kg}$.

\section{DISCUSSION}

With more and more couples opting for the benefits of in vitro fertilisation today, the incidence of heterotopic pregnancies has seen a steady rise. The incidence of heterotopic pregnancies occurring in the cornual region is even rarer. Cornual pregnancy occurs when the embryo implants in the intramural portion of the fallopian tube. ${ }^{3}$ Diagnosis of cornual heterotopic pregnancy is often delayed, with only $10 \%$ of cases reported in the literature diagnosed before operative intervention. ${ }^{4}$ bHcg levels are not useful as they may be already raised due to presence of intrauterine gestation. Also, the presence of cornual pregnancy may be missed, once intrauterine gestation is detected. So, a high index of suspicion is required to anticipate and diagnose the condition which may lead to a catastrophic outcome if not managed timely.

Various factors predispose to cornual heterotopic pregnancies. In ART cycles, there is evidence to suggest that increased number of embryos transferred, increases multiple gestation rates and predisposes toward an increased risk of heterotopic pregnancy. ${ }^{5}$ Pre-existing tubal pathologies such as hydrosalpinx and proximal tubal occlusion had higher rates of ectopic pregnancy and heterotopic pregnancy. ${ }^{6}$ Some authors advocate the removal of tubes with hydrosalpinx before IVF cycles as it predisposes to low conception rates due to embyotoxic factors and ectopic pregnancies. Bilateral salpingectomy does not prevent the development of a cornual pregnancy, however, and it may, in fact increases the risk of cornual pregnancy after use of assisted reproductive technology. ${ }^{7}$ In our case also, the heterotopic pregnancy developed at cornu on the site of previous salpingectomy.

Several mechanisms unique to embryo transfer procedures in IVF, may predispose to a heterotopic pregnancy: positioning of the tip of the embryo transfer catheter toward the tubal ostia at the time of expulsion of the embryo; retrograde delivery of the embryos because of reflux of uterine secretions, uterine contractions or endometrial bleeding from traumatic embryo transfer; extrusion of the embryo into the cornual or tubal area by hydrostatic forces involved in the transfer and a greater volume of fluid loaded in the embryo transfer catheter. ${ }^{7}$ We highly advocate the placement of a stitch at the cornual end in cases of salpingectomies to decrease cornual ectopics from happening.

The initial modality of detection of a cornual heterotopic pregnancy is ultrasound. Transvaginal ultrasound is better than transabdominal modality. The ultrasonographic criteria for diagnosis include - a wide bridge of myometrium separating the gestations, a chorionic sac seen separately and more than $1 \mathrm{~cm}$ from the most lateral edge of the uterine cavity with a thin myometrial layer surrounding the chorionic sac. ${ }^{8}$ MRI can be confirmatory in doubtful cases.

The management of cornual heterotopic pregnancy may vary from case to case. In the absence of fetal cardiac activity, with early diagnosis in the first trimester, a symptom-free patient, limited craniocaudal length and small gestational sac size, expectant management can be an option. ${ }^{9}$

In patients with stable vitals and the condition accidentally detected on ultrasound, KCL administration into the cornual pregnancy can selectively reduce it. A scoring system was introduced by Fernandez et al to evaluate the success of non- surgical management of heterotopic cornual pregnancy. Six criteria were studied: gestational sac, $\beta$-human chorionic gonadotropin level, progesterone level, presence of abdominal pain, volume of haemoperitoneum, and the ultrasonographic diameter of the hematosalpinx. If the total score was $\leq 12$, then the likelihood of success of medical treatment would be > $80 \% .{ }^{10}$ The main advantage of nonsurgical treatment is that it is less invasive and inexpensive, with quick recovery, minimal blood loss, and a short length of hospital stay compared to surgical treatment. However, nonsurgical treatment has several concerns such as a possible cornual rupture associated with ultrasoundguided puncture and massive bleeding. ${ }^{3}$

However, patients presenting with a ruptured cornual pregnancy, haemodynamic status of the patient decides the approach towards management. In cornual heterotopic pregnancies, the incidence of rupture may be as high as $48.6 \% .^{7}$ This is primarily due to the fact that the cornu is a highly vascular structure being supplied by the branches of the ovarian as well as the uterine arteries.

In patients with minimal haemoperitoneum, laparoscopic surgery gives good results, giving benefit of minimally invasive surgery to the patient. The advantages of laparoscopic management over exploratory laparotomy are the shorter hospital stay, fewer surgical wounds, and reduced use of antibiotics and analgesics. ${ }^{3}$

Laparotomy is the mainstay of management in many patients as they present with a ruptured cornual pregnancy with haemoperitoneum. Grobman et al recommended excision in larger gestations. ${ }^{11}$ In the review by Habbana et al it was found that the laparotomy group had high live birth rates. ${ }^{7}$ Surgery, however, is 
made challenging by the fact that the tissues are friable, there is increased vascularity, there is difficulty in controlling bleeding. These have to be balanced against the risk of jeopardizing the intrauterine gestation. In case the bleeding is not controlled, one may even have to resort to hysterectomy in such cases.

\section{CONCLUSION}

Considering the rare occurrence of heterotopic cornual pregnancy, there is not consensus over its management. Also, the management options need to be individualised so that an effort can be made to preserve the very precious intrauterine pregnancy. The dilemmas, the associated medico legal issues, delayed clinical presentation, varied management approaches, careful monitoring required of the remaining intrauterine pregnancy and possibility of uterine rupture at the cornual site during the growth of remaining pregnancy make cornual heterotopic pregnancy a challenge for clinicians. However, if judiciously managed, an obstetric crisis can be averted with a favourable surgical outcome and a high take home baby rate can be achieved.

Funding: No funding sources

Conflict of interest: None declared

Ethical approval: Not required

\section{REFERENCES}

1. Devoe R, Pratt J. Simultaneous intrauterine and extrauterine pregnancy. Am J Obstet Gynecol. 1948;56:1119-26.

2. Ikeda SI, Sumiyoshi M, Nakae M, Tanaka S, Ijyuin $\mathrm{H}$. Heterotopic pregnancy after in vitro fertilization and embryo transfer. Acta Obstet Gynecol Scand. 1998;77(4):463-4.

3. Kim MJ, Jung YW, Cha JH, Seok HH, Han JE, Seong SJ, et al. Successful management of heterotopic cornual pregnancy with laparoscopic cornual resection. Eu J Obstet Gynecol Repro Biol. 2016;203:199-203.
4. Fernandez H, Lelaidier C, Doumerc S, Fournet P, Oliveness F, Frydman R. Nonsurgical treatment of heterotopic pregnancy:a report of six cases. Fertil Steril. 1993;60:428-32.

5. Chen SU, Yang YS, Ho HN, Ko TM, Hsieh FJ, Lee TY. Combined cornual pregnancy and intrauterine twin pregnancy after in vitro fertilization and embryo transfer: report of a case. J Formos Med Assoc. 1992;91:1002-5.

6. Dubuisson J, Aubriot F, Mathieu L, Foulot H, Mandelbrot L, de Joliniere B. Risk factors for ectopic pregnancy in 556 pregnancies after in vitro fertilization: implications for preventive management. Fertil Steril. 1995;56:686-90.

7. Habana A, Dokras A, Giraldo JL, Jones EE. Cornual heterotopic pregnancy: contemporary management options. Am J Obstet Gynecol. 2000;182(5):1264-70.

8. Timor-Tritsch I, Monteagudo A, Matera C, Veit C. Sonographic evolution of cornual pregnancies treated without surgery. Obstet Gynecol. 1992;79:1044-9.

9. Sentilhes L, Bouet PE, Gromez A, Poilblanc M, Lefebvre-Lacoeuille C, Descamps P. Successful expectant management for a cornual heterotopic pregnancy. Fertil Steril. 2009;91:e11-e13.

10. Fernandez H, Lelaidier C, Doumerc S, Fournet P, Oliveness F, Frydman R. Nonsurgical treatment of heterotopic pregnancy: a report of six cases. Fertil Steril. 1993;60:428-32.

11. Grobman WA, Milad MP. Conservative laparoscopic management of a large cornual ectopic pregnancy. Hum Reprod. 1998;13:2002-4.

Cite this article as: Bansal V, Chhabra M, Chopra R, Prajapati P. Successful live birth after heterotopic ruptured cornual pregnancy with twin intrauterine gestation in an in vitro fertilization conception. Int $\mathbf{J}$ Reprod Contracept Obstet Gynecol 2019;8:5057-61. 